Check for updates

Cite this: RSC Adv., 2017, 7, 56359

Received 3rd November 2017

Accepted 27th November 2017

DOI: $10.1039 / c 7 r a 12098 b$

rsc.li/rsc-advances

\section{A fluorescence switch sensor for detection of virginiamycin based on graphene oxide-supported carbon quantum dots and molecularly imprinted polymer}

\begin{abstract}
Shuhuai Li, (D)*ab Jianping Li, (D) ${ }^{c}$ Jinhui Luo, ${ }^{\text {ab }}$ Qun Zhang $^{\mathrm{ab}}$ and Lianming Zhang ${ }^{\mathrm{c}}$
A fluorescence switch sensor was developed for the detection of virginiamycin, which was based on graphene oxide-supported carbon quantum dots (GO/C-dots) as the signal indicator and molecularly imprinted polymer (MIP) as the recognition template. First, GO/C-dots were prepared on the surface of an indium tin oxide (ITO) electrode. Then, MIP with virginiamycin as the template molecule and oaminophenol as the functional monomer were synthesized on the surface of the GO/C-dots-modified ITO electrode. After removal of virginiamycin from the MIP, a sensor for specific adsorption of virginiamycin was obtained. The GO/C-dots generated a bright fluorescent signal, while virginiamycin quenched this fluorescence. Therefore, elution and adsorption of virginiamycin by the MIP acted as a switch to control fluorescence intensity. The obtained sensor has a high sensitivity and good selectivity, with a detection limit of $1.56 \times 10^{-11} \mathrm{~mol} \mathrm{~L}^{-1}$ for virginiamycin.
\end{abstract}

\section{Introduction}

Residual antibiotics in animal products are harmful for human life and health. Therefore, the detection and monitoring of residual antibiotics has attracted increasing attention. Virginiamycin $^{1}$ is a type of antibiotic that has been widely used for the treatment of bacterial infections in animals because of its good spectrum of activity against both Gram-positive and Gramnegative bacteria. The residual virginiamycin in meat products and the environment is a huge potential danger for public health. Typically, residual antibiotics are detected by highpressure liquid chromatography (HPLC) ${ }^{2}$ and HPLC-tandem mass spectrometry (HPLC-MS). ${ }^{3}$ However, traditional measurement methods involve high costs of operation and require complex pre-treatment of samples. Therefore, it is necessary to develop a sensitive, selective, inexpensive, and simple method for the detection of residual antibiotics. Due to the excellent selectivity, sensors-based on molecularly imprinted polymer (MIP) have found wide application. ${ }^{4,5}$

Molecular imprinting technique ${ }^{6}$ is a new and effective separation process that can prepare MIPs, ${ }^{7}$ featuring selective recognition of a specific molecule. Thus, an MIP can be selected as the recognition template for sensor preparation. Due to

${ }^{a}$ Analysis and Test Center of Chinese Academy of Tropical Agricultural Sciences, Haikou, 571101, China.E-mail: happylishuhuai@163.com; luocatas@21cn.com ${ }^{b}$ Laboratory of Quality \& Safety Risk Assessment for Tropical Products (Haikou), Ministry of Agriculture, Haikou, 571101, China

${ }^{c}$ College of Chemistry and Bioengineering, Guilin University of Technology, Guilin, 541004, China
MIP's outstanding molecular recognition features and its excellent selectivity to substrates, MIP-based sensors are widely applied in analytical chemistry applications, including veterinary drug testing, ${ }^{8}$ environmental protection, ${ }^{9}$ and life sciences. ${ }^{\mathbf{1 0}}$ Among numerous types of sensors, fluorescence sensors ${ }^{11,12}$ based on MIPs have received considerable attention due to several advantages, such as high sensitivity, selectivity, and feasibility of fabrication. Thus, the florescent sensors have been rapidly developed for the detection of residual veterinary antibiotics ${ }^{13}$ and pesticides. ${ }^{14}$ Hou et $a l .{ }^{15}$ developed a fluorescence sensor for the detection of tetracycline by grafting imprinted polymers onto the surface of carbon quantum dots. This approach was combined the highly selective adsorption properties of MIPs with the sensitivity of fluorescence detection. In terms of improving the sensitivity of fluorescence sensors, recent strategies have been focused on developing nanometresized materials to amplify the detected signal. Graphene ${ }^{16}$ is a honeycomb-like sheet of carbon of only one atom thickness with a number of unique physical properties, such as a very large specific surface area, excellent thermal stability, and excellent optical performance. It is, therefore, being used as the base material for signal amplification in fluorescence sensors. ${ }^{17,18}$ Carbon dots (C-dots), ${ }^{19}$ likewise, are a new class of fluorescent nanomaterials with attractive features, such as low cytotoxicity, good biocompatibility, and tuneable excitation and emission wavelengths. The development of composite graphene-supported C-dots can therefore have great potential for increasing the sensitivity and stability of fluorescent sensors. To our knowledge, however, the use of graphene oxide- 


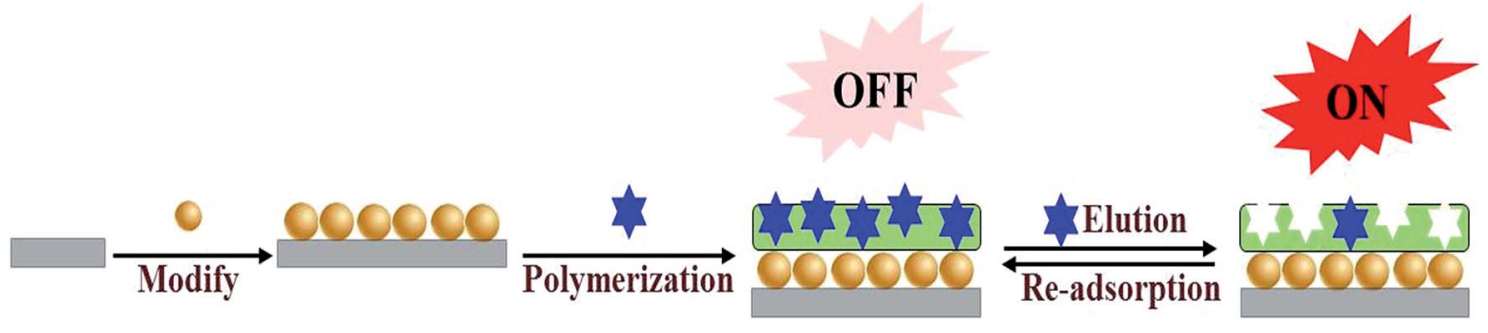

\section{ITO $\odot$ GO/C-dots $\downarrow$ Virginiamycin $\square$ MIP}

Scheme 1 Fabrication of the switch sensor for the detection of virginiamycin.

supported C-dots (GO/C-dots) together with an MIP sensor for virginiamycin detection has not been reported.

In this study, virginiamycin was selected as the analysis object and an MIP fluorescent switch sensor was fabricated for its selective detection. Initially, GO/C-dots were prepared by a hydrothermal method and coated onto indium tin oxide (ITO) electrodes. Subsequently, MIP was prepared via electropolymerisation using $o$-aminophenol as functional monomer and virginiamycin as the template molecule. After removal of the template molecule, the MIP sensors displayed stereo cavities in the MIP for recognizing (binding) virginiamycin. When a cavity was empty, the bright fluorescence of GO/C-dots was detected; in this conformation, the switch was "ON". When virginiamycin was adsorbed onto the MIP, the fluorescence intensity of the GO/C-dots decreased; in this conformation, the switch was "OFF". Thus, this switch controlled the fluorescence intensity of the GO/C-dots in the MIP by eluting and adsorbing virginiamycin as shown in Scheme 1. In addition, the detection signal was amplified by GO. Thus, a new method based on a fluorescence switch sensor for virginiamycin detection was established; this sensor exhibited high linearity, stability, sensitivity, and resolution, and can be applied for the detection of virginiamycin in real meat samples.

\section{Experimental}

\section{Apparatus and reagents}

Fluorescence spectra were performed on an F-2500 photospectrometer (Hitachi, Tokyo, Japan). Electrochemical measurements were performed using an electrochemical workstation (CHI660D, Beijing Huake Putian Technology Co., Ltd., Beijing, China). The classical three-electrode system consisted of a $\mathrm{KCl}$-saturated $\mathrm{Ag} / \mathrm{AgCl}$ electrode as the reference electrode, a platinum wire electrode as the auxiliary electrode, and an MIP-modified ITO electrode as the working electrode. Transmission electron microscope (TEM) images were acquired using a Tecnai 30F TEM (Philips Electronics N.V., Amsterdam, Netherlands). X-ray photoelectron spectroscopy (XPS) was carried out using a VG Multilab 2000 spectrometer (Thermo Electron Corporation, USA). The diameter distribution analysis of NPs was performed using a ZS90 Nano particle size and zeta potential analyser (Malvern co., LTD, England).

Virginiamycin, gentamicin, amoxicillin, lincomycin, oxytetracycline, azithromycin, kanamycin, streptomycin, cefquinome, and tetracycline standards were bought from Dr Ehrenstorfer GmbH, Germany (Augsburg, Germany). The o-phenylenediamine was obtained from Xilong Scientific Co., Ltd, China (Shantou, China). GO was bought from Nanjing XFNANO Materials Tech Co., Ltd, China (Nanjing, China). All reagents used in our experiments were of analytical grade and all aqueous solutions were prepared with doubly distilled water.

\section{Preparation of GO/C-dots modified ITO electrode}

C-dots were synthesized using a hydrothermal method as reported in ref. 20. Briefly, sodium citrate $(0.30 \mathrm{~g})$ and $\mathrm{NH}_{4} \mathrm{HCO}_{3}$ $(2.4 \mathrm{~g})$ and $20 \mathrm{~mL}$ ultrapure water were added in a reaction vessel, which was sealed inside a Teflon lined stainless steel autoclave and heated at $200{ }^{\circ} \mathrm{C}$ for $3 \mathrm{~h}$, following which the autoclave was allowed to cool to room temperature. Purification of the obtained C-dots was carried out using dialysis tubing. Subsequently, $0.10 \mathrm{~g}$ GO and $0.10 \mathrm{~g}$ C-dots were dissolved in $10 \mathrm{~mL}$ Tris- $\mathrm{HCl}$ (0.05 mol L $\left.{ }^{-1}, \mathrm{pH} 7.6\right)$; after ultrasonic treatment for $30 \mathrm{~min}, \mathrm{GO} / \mathrm{C}$-dots were obtained. The ITO electrode was cleaned via ultrasonication in acetone, ethanol, and double-distilled water for $5 \mathrm{~min}$ each. Finally, the ITO electrode was soaked in $20 \mu \mathrm{L} \mathrm{GO} / \mathrm{C}$-dots for $5 \mathrm{~min}$ and dried at $50{ }^{\circ} \mathrm{C}$.

\section{MIP-modified ITO electrode sensor fabrication}

MIP and non-MIP electrodes (nMIP) were prepared directly on a GO/C-dots-modified ITO electrodes via electropolymerisation in $10 \mathrm{~mL}$ PBS $\left(0.05 \mathrm{~mol} \mathrm{~L}^{-1}, \mathrm{pH} 7.4\right.$, contained $3 \times 10^{-4} \mathrm{~mol} \mathrm{~L}^{-1}$ $o$-aminophenol and $1 \times 10^{-4} \mathrm{~mol} \mathrm{~L}^{-1}$ virginiamycin). The MIP was formed on an ITO substrate after 20 cycles of cyclic voltammetry $(\mathrm{CV})$ in the polymer solution by sweeping the potential in the range from 0 to $+1.0 \mathrm{~V}$ at a scan rate of $50 \mathrm{mV} \mathrm{s}^{-1}$. After electropolymerisation, the modified ITO electrode was washed with methanol for $10 \mathrm{~min}$ to remove the imprinting molecules. The nMIP sensor was fabricated using the same method as above, but without the use of virginiamycin. 


\section{Electrochemical and fluorescence measurements}

Electrochemical experiments were performed in a $3 \times$ $10^{-4} \mathrm{~mol} \mathrm{~L}^{-1} \quad \mathrm{~K}_{3}\left[\mathrm{Fe}(\mathrm{CN})_{6}\right] / \mathrm{K}_{4}\left[\mathrm{Fe}(\mathrm{CN})_{6}\right]$ solution containing $0.5 \mathrm{~mol} \mathrm{~L}^{-1} \mathrm{KCl}$. $\mathrm{CV}$ was performed over the potential range from 0 to $+0.8 \mathrm{~V}$. Electrochemical impedance spectroscopy (EIS) was performed over the frequency range of 0.1 to $100000 \mathrm{~Hz}$ by applying an alternating voltage of $5 \mathrm{mV}$. Fluorescence spectra were measured over the wavelength range of $300-700 \mathrm{~nm}$ at an excitation wavelength of $260 \mathrm{~nm}$ in the Tris-HCl buffer (0.05 $\left.\mathrm{mol} \mathrm{L}^{-1}, \mathrm{pH} 7.6\right)$. The excitation and emission slits of the instrument were set to a width of $3 \mathrm{~nm}$.

\section{Sample treatment}

Virginiamycin was extracted from meat samples via ultrasonic treatment. Briefly, $20 \mathrm{~mL}$ of acetonitrile was add to a $5.0 \mathrm{~g}$ meat sample and ultrasonicated for $30 \mathrm{~min}$, then transferred into a $50 \mathrm{~mL}$ flask for evaporation and dried using a rotary evaporator. The final residue was dissolved in $1.0 \mathrm{~mL}$ of methanol.

\section{Results and discussion}

\section{Characterization of GO/C-dots}

The morphology of the GO/C-dots nanocomposite was characterized using a TEM. As shown in Fig. 1A, GO showed a thin flake structure with nanometer thickness. The sliced edges of GO were wrinkled, which could reduce the free energy of the system. C-dots were evenly distributed across the entire surface of GO as shown in Fig. 1B. Moreover, the C-dots were predominantly spheroidal particles with nanometer size. The size distribution of C-dots was investigated using a particle size analyser. As shown in Fig. 1C, the sizes of C-dots are primarily in the range of 8-40 $\mathrm{nm}$ with an average diameter of $21 \mathrm{~nm}$. The predominant elements in GO/C-dots were analysed by XPS. Since the entire synthesis process was very simple and avoids
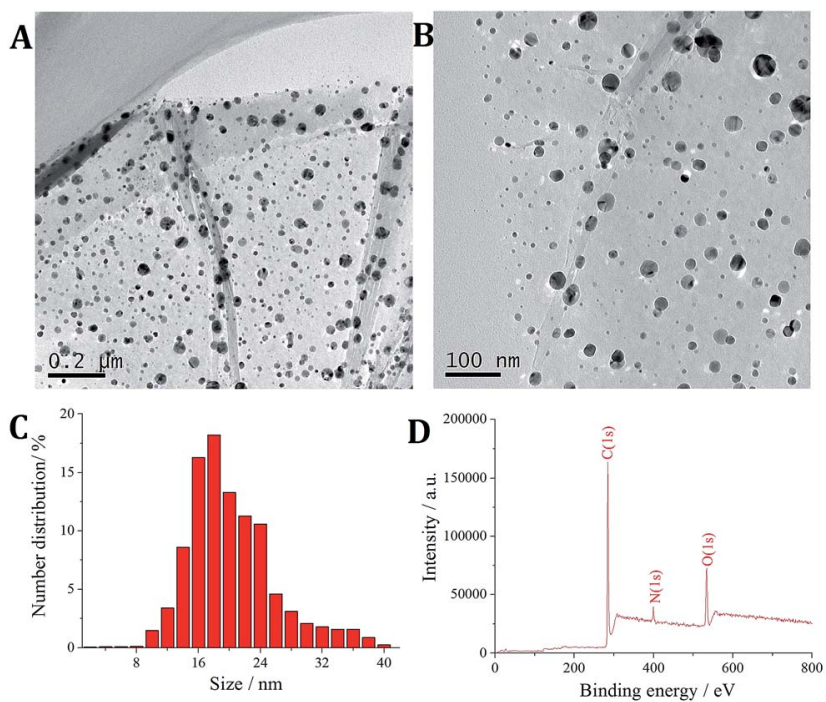

Fig. 1 (A) TEM images of GO/C-dots; (B) TEM images of C-dots; (C) particle size distribution of C-dots; (D) XPS of GO/C-dots. the superfluous impurities, the GO/C-dots nanocomposites primarily composed of C, O, and N. As shown in Fig. 2D, C (1s) at $284 \mathrm{eV}$ primarily originated from GO and the C-dots, $\mathrm{O}(1 \mathrm{~s})$ at $530 \mathrm{eV}$ primarily originated from GO, and N (1s) at $399 \mathrm{eV}$ primarily originated from chemical groups on the surface of the C-dots. Thus, based on these results, we infer that the $\mathrm{GO} / \mathrm{C}$ dots nanocomposite was successfully synthesized.

\section{Evaluation of the MIP}

$\mathrm{K}_{3}\left[\mathrm{Fe}(\mathrm{CN})_{6}\right] / \mathrm{K}_{4}\left[\mathrm{Fe}(\mathrm{CN})_{6}\right]$ was used as the redox probe to characterize MIP film formation on glassy carbon electrode (GCE) through CV and EIS methods. Although $o$-aminophenol film is nonconductive, cavities formed in the MIP after removal of virginiamycin can act as channels for electron transport. Thus, binding of the virginiamycin in the cavities can result in a corresponding change in redox current. As shown in Fig. 2A and B, ITO is a good conductor of electricity; there was a remarkable redox current (Fig. 2A, curve a) and small resistance (Fig. 2B, curve a) when the GO/C-dots were modified on the ITO. The peak current of the probe decreased (Fig. 2A, curve b), while the resistance increased (Fig. 2B, curve b). For MIP formed on the surface of ITO, it was difficult for the redox probe to react on ITO, causing a sharp decrease in the peak current of the probe (Fig. 2A, curve c) and a sharp increase in resistance (Fig. 2B, curve c). After virginiamycin removal, the cavities in MIP functioned as channels for electron transport and the peak current of the probe increased (Fig. 2A, curve d) and the resistance decreased (Fig. 3B, curve d). Upon virginiamycin re-adsorption in the MIP, the cavities were blocked and the channels for probe entrance disappeared again, resulting in a decrease in peak current (Fig. 2A, curve e) and increase in resistance (Fig. 2B, curve e). For nMIP, on the other hand, no channels for electron transport existed, so the peak current of the probe barely changed before and after nMIP modified ITO electrode was washed with methanol (Fig. 2A curve f and curve g). We
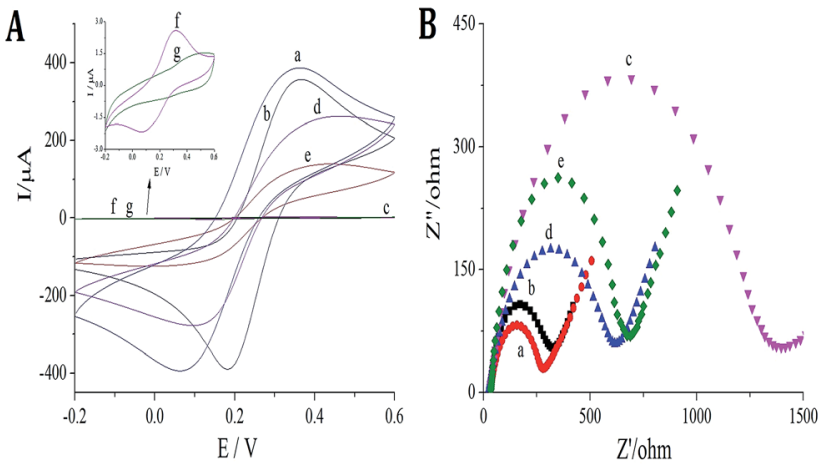

Fig. 2 Characterization of MIP films: (A) CVs: (a) ITO bare electrode; (b) GO/C-dots modified ITO electrode; (c) MIP modified ITO electrode; (d) MIP modified ITO electrode after virginiamycin removal; (e) MIP modified ITO electrode after re-adsorption of $1 \times 10^{-8} \mathrm{~mol} \mathrm{~L}^{-1}$ virginiamycin; (f) nMIP modified ITO electrode; (g) nMIP modified ITO electrode washed with methanol; (B) (a) ITO bare electrode; (b) GO/Cdots modified ITO electrode; (c) MIP modified ITO electrode; (d) MIP modified ITO electrode after virginiamycin removal; (e) MIP modified ITO electrode after re-adsorption of $1 \times 10^{-8} \mathrm{~mol} \mathrm{~L}^{-1}$ virginiamycin. 


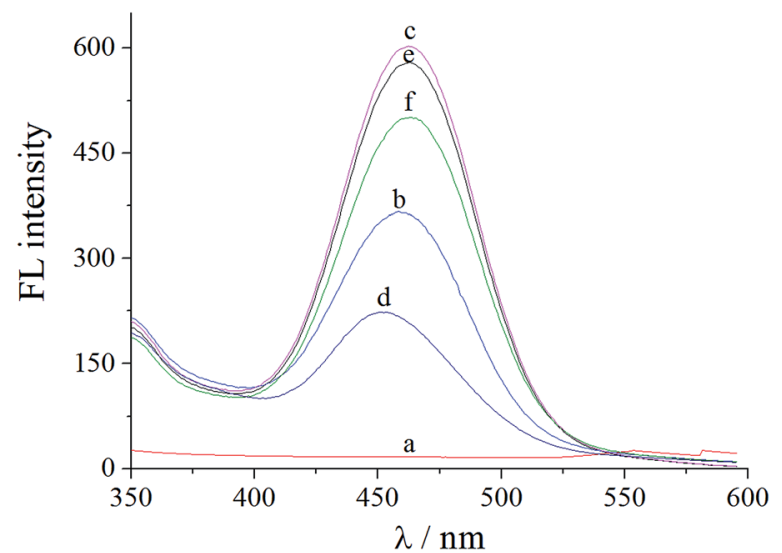

Fig. 3 Fluorescence response of sensors: (a) bare ITO electrode; (b) C-dots modified ITO electrode; (c) GO/C-dots modified ITO electrode; (d) MIP modified ITO electrode; (e) MIP modified ITO electrode after virginiamycin removal; (f) MIP modified ITO electrode after readsorption of $1 \times 10^{-8} \mathrm{~mol} \mathrm{~L}^{-1}$ virginiamycin.

hypothesize that some cavities were deformed, which made it difficult for template molecules to enter the cavities. Nonetheless, from the above results, it is evident that the molecularly imprinted membrane showed a clear response to virginiamycin.

\section{Fluorescence of the MIP sensor}

The sensor offered a switch to control the fluorescence of C-dots by virginiamycin removal and re-adsorption. As shown in Fig. 3, no fluorescence signal was detected for ITO (curve a), while for the C-dots modified on the surface of ITO, a visible fluorescence signal at $560 \mathrm{~nm}$ (curve b) was observed, which was significantly enhanced by GO (curve c). The latter resulted from the excellent electrical conductivity and high specific surface area of GO. Moreover, GO has excellent optical properties and facilitates transmission of photons. After MIP was prepared on the GO/Cdots-modified ITO, the fluorescence intensity significantly decreased due to fluorescence quenching of the $\mathrm{GO} / \mathrm{C}$-dots (curve d) by virginiamycin. After removal of virginiamycin MIP, the switch turned "ON" and the fluorescence intensity

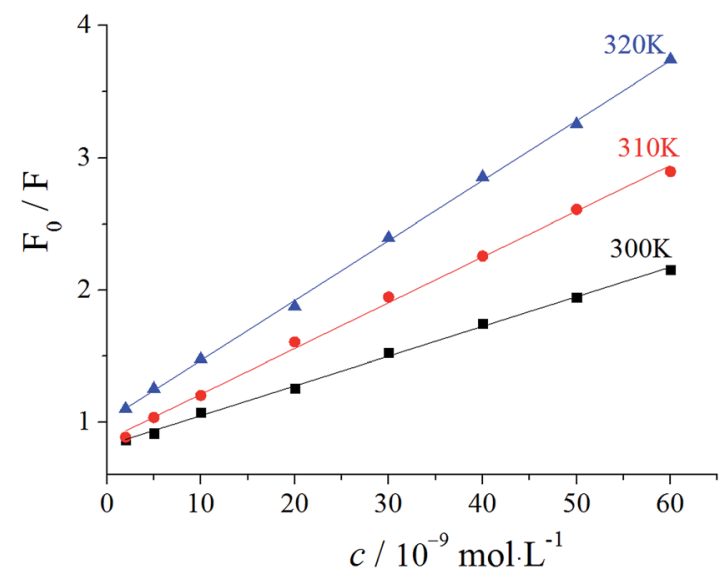

Fig. 4 Effect of temperature on fluorescence intensity of GO/C-dots.
Table 1 Parameters of Stern-Volmer plots of GO/C-dots

\begin{tabular}{llll}
\hline Temperature/K & $\begin{array}{l}\left(K S_{\mathrm{b}} \times 10^{5}\right) / \\
\left.(\mathrm{L} \mathrm{mol})^{-1}\right)\end{array}$ & $\begin{array}{l}\text { Stern-Volmer linear } \\
\text { equation }\end{array}$ & $R$ \\
\hline 300 & 0.0454 & $F_{0} / F=0.0454 c+1.013$ & 0.9991 \\
310 & 0.0346 & $F_{0} / F=0.0346 c+0.864$ & 0.9988 \\
320 & 0.0225 & $F_{0} / F=0.0225 c+0.824$ & 0.9997
\end{tabular}

recovered (curve e). When virginiamycin was re-adsorbed onto MIP, the switch turned "OFF" and the fluorescence quenched again (curve f). The involved mechanism could be dominated by dynamic quenching processes. To substantiate the mechanism involved, the relation between virginiamycin concentration and fluorescence intensity of the GO/C-dots was studied using the Stern-Volmer equation. The results are shown in Fig. 4 and Table 1. The fluorescence quenching constant increased with increase in temperature and the quenching constant was much lower than $200 \mathrm{~L} \mathrm{~mol}^{-1}$. These results indicate that the kinetics of the quenching reacdtions correspond to dynamic quenching processes. ${ }^{21}$ Excited electrons of the C-dots can enter conduction bands, forming electron-hole pairs. When these electrons are captured by virginiamycin, electron-hole pairs can be destroyed, resulting in fluorescence quenching.

\section{Optimization of method}

The experimental conditions were optimized for virginiamycin detection, including the time for template removal, time for readsorption, and $\mathrm{pH}$ of the buffer. As shown in Fig. 5A, when the template was removed from the MIP, the fluorescence intensity increased. The fluorescence intensity was measured every minute; it initially increased gradually and then remained constant after $5 \mathrm{~min}$ (curve a). When $8 \times 10^{-8} \mathrm{~mol} \mathrm{~L}^{-1}$ virginiamycin was re-adsorbed onto the MIP, the switch turned "OFF", causing a decrease in signal.

Furthermore, the fluorescence intensity was measured every minute; it initially decreased gradually and then remained constant after 6 min (curve b). Thus, the optimum time for virginiamycin removal and re-adsorption was 5 and $6 \mathrm{~min}$, respectively. The effects of $\mathrm{pH}$ were investigated after re-
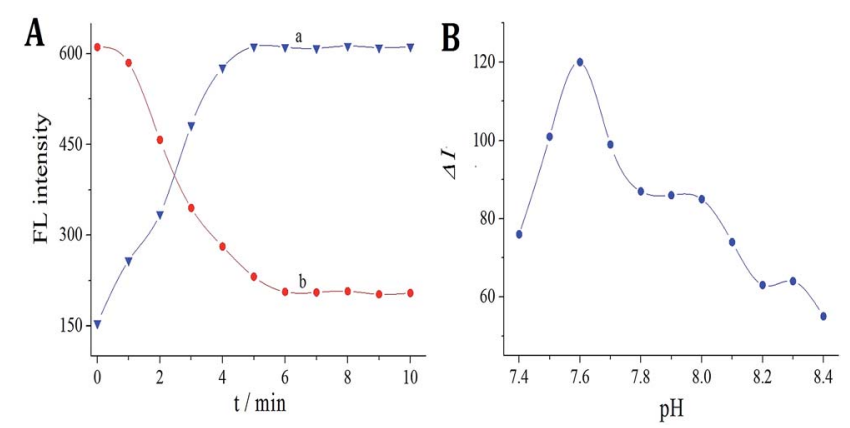

Fig. 5 (A) (a) Effects of template removal on FL intensity; (b) effects of re-adsorption on $8 \times 10^{-8} \mathrm{~mol} \mathrm{~L}^{-1}$ virginiamycin. (B) Effects of $\mathrm{pH}$ on 1 $\times 10^{-8} \mathrm{~mol} \mathrm{~L}^{-1}$ virginiamycin. 


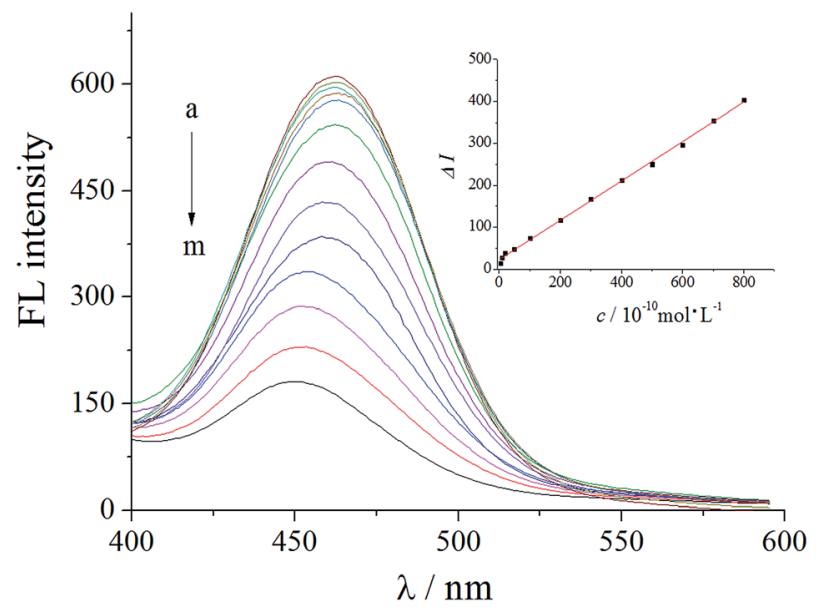

Fig. 6 Fluorescent response of the sensor with adsorbed virginiamycin at different concentrations. (a-m) $(0,5,10,20,50,100,200$, $300,400,500,600,700$, and 800$) \times 10^{-10} \mathrm{~mol} \mathrm{~L}^{-1}$ virginiamycin.
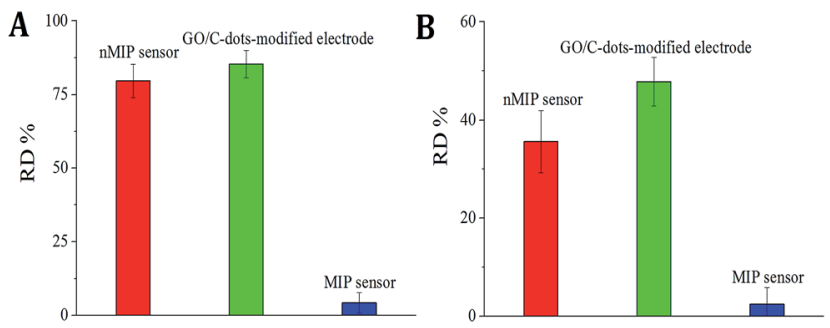

Fig. 7 (A) RDs of sensors with or without antibiotics interference; (B) RDs of sensors with or without interference of coexisting ions.

adsorption of $8 \times 10^{-8} \mathrm{~mol} \mathrm{~L}^{-1}$ virginiamycin in the $\mathrm{pH}$ range from 7.4 to 8.2 (Fig. 5B); the results reveal that the fluorescence quenching intensity reached a maximum at $\mathrm{pH}$ 7.6, which was therefore selected as the optimum $\mathrm{pH}$.

\section{Calibration curve}

Fluorescence was measured after treatment of the developed sensor with different concentrations of virginiamycin. From the experimental results, it was found that the relationship between the concentration of virginiamycin $(c)$ and the decrease in the fluorescence intensity $(\Delta I)$ was linear in the range from $5.0 \times$ $10^{-10}$ to $8.0 \times 10^{-8} \mathrm{~mol} \mathrm{~L}^{-1}$. As shown in Fig. 6, the linear regression fit was $\Delta I=0.47 c\left(10^{-10} \mathrm{~mol} \mathrm{~L}^{-1}\right)+22.3$, with a correlation coefficient $(r)$ of 0.9992 . The detection limit (DL) was $1.56 \times 10^{-11} \mathrm{~mol} \mathrm{~L}^{-1}$ using the formula $\mathrm{DL}=K S_{\mathrm{b}} / a$, where $a$ represents the slope of the calibration plot, $S_{\mathrm{b}}$ represents the standard deviation of the replicate blank measurements, and $K$ is a constant with value 3 . Compared with other methods in the literature, the sensor with GO/C-dots was more sensitive and had a lower detection limit.

\section{Selectivity of the MIP sensor}

The selectivity of MIP sensors typically relies on the MIP recognition sites. Therefore, the nMIP sensor and GO/C-dotsmodified electrode was compared with the MIP sensor. The adsorption procedures were performed as described above using commercial antibiotics such as gentamicin, amoxicillin, lincomycin, oxytetracycline, azithromycin, kanamycin, streptomycin, cefquinome, and tetracycline in $5 \times 10^{-9} \mathrm{~mol} \mathrm{~L}^{-1}$ virginiamycin; the resultant fluorescence intensities were studied. As shown in Fig. 7A, no difference was observed after virginiamycin re-adsorption on the MIP sensor between solutions with or without a mixture of the above-mentioned antibiotics. However, fluorescence tests showed that there were visible changes for the nMIP sensor (the relative deviation [RD] was 79.6\%) and GO/C-dots-modified electrode $(\mathrm{RD}=85.3 \%)$ after addition of the above-mentioned pesticides. This occurred because the nMIP sensor and GO/C-dots-modified electrode did not have specific recognition sites for virginiamycin. In addition, interference with the detection of virginiamycin by coexisting ions was also tested. As shown in Fig. 7B, the fluorescence intensity for the MIP sensor was slightly altered (RD $<5.0 \%$ ) using solutions with an added mixture of 2000-fold (relative to the concentration of virginiamycin) $\mathrm{Cu}^{2+}, \mathrm{Cd}^{2+}, \mathrm{Fe}^{2+}, \mathrm{CO}_{3}{ }^{2-}$, $\mathrm{Pd}^{2+}, \mathrm{Na}^{+}, \mathrm{K}^{+}, \mathrm{Zn}^{2+}, \mathrm{SO}_{3}{ }^{2-}, \mathrm{NO}^{3-}, \mathrm{PO}_{4}{ }^{3-}, \mathrm{SO}_{4}{ }^{2-}, \mathrm{Hg}^{2+}, \mathrm{Cl}^{-}, \mathrm{F}^{-}$, $\mathrm{Mg}^{2+}, \mathrm{NH}_{4}^{+}, \mathrm{I}^{-}, \mathrm{Fe}^{3+}, \mathrm{Cr}^{3+}, \mathrm{Br}^{-}, \mathrm{Ni}^{2+}, \mathrm{Ca}^{2+}$, and $\mathrm{Mn}^{2+}$. However, the $\mathrm{RD}$ values for the nMIP sensor and GO/C-dots-modified electrode were 35.6 and $47.8 \%$, respectively. These results strongly suggest that the MIP sensor exhibited good selectivity for virginiamycin.

\section{Reproducibility and stability of the sensor}

The reproducibility and stability of the sensor were studied by measuring the fluorescence intensities. A relative standard deviation (RSD) of $2.8 \%$ was obtained by measurement of the fluorescence intensities for five different sensors, which indicated good sensor-to-sensor reproducibility. In addition, an RSD of $4.7 \%$ was obtained by measurement of the fluorescence intensities using the same sensor in five measurements. To test

Table 2 Results of sample assay and recoveries

\begin{tabular}{|c|c|c|c|c|c|c|c|}
\hline Samples & $\begin{array}{l}\text { This method } \\
(n=5)\end{array}$ & RSD\% & $\begin{array}{l}\text { HPLC-MS method } 10^{-10} \\
\operatorname{mol~L}^{-1}(n=5)\end{array}$ & $\begin{array}{l}\text { Added } 10^{-10} \\
\text { mol L }^{-1}\end{array}$ & $\begin{array}{l}\text { Total found } 10^{-10} \\
\operatorname{mol~L}^{-1}(n=5)\end{array}$ & $\mathrm{RSD} \%$ & Recoveries\% \\
\hline Chicken & 7.80 & 2.80 & 7.75 & 10.00 & 16.54 & 3.21 & 87.4 \\
\hline Beef & Not detected & - & Not detected & 10.00 & 9.24 & 4.32 & 92.4 \\
\hline Pork & Not detected & - & Not detected & 15.00 & 16.10 & 3.68 & 107.3 \\
\hline Mutton & Not detected & - & Not detected & 15.00 & 14.32 & 4.98 & 95.5 \\
\hline Carp & Not detected & - & Not detected & 20.00 & 17.85 & 3.77 & 89.2 \\
\hline
\end{tabular}


its stability, the sensor was dipped in double distilled water at $4{ }^{\circ} \mathrm{C}$ when not in use. The fluorescence response detected every two days remained nearly unchanged for 10 days. After a month, the fluorescence intensity decreased by about $10.8 \%$ as compared with the initial response. This indicated that the sensor exhibited good reproducibility and stability.

\section{Sample detection}

The proposed method was next applied to the detection of virginiamycin in actual meat samples. Five types of animal products (chicken, beef, pork, mutton, and carp) were obtained from a wholesale market (West Market in Haikou City of China) and pre-treated according to the procedure outlined in Section "Sample treatment". The detection results using our asfabricated MIP sensor were compared with the results obtained from HPLC-MS analysis using the same samples. As shown in Table 2, recoveries between 87.4 and $107.3 \%$ were observed. These results demonstrate that the developed sensor could be used to analyse the amount of virginiamycin in real samples.

\section{Conclusions}

In summary, a fluorescence switch sensor using a molecularly imprinted polymer (MIP) as the recognition template and graphene oxide-modified carbon dots (GO/C-dots) as the signal source was developed for virginiamycin determination. The fabrication of the MIP and GO/C-dots system offers several advantages, such as high sensitivity and selectivity, which facilitate anti-interference for the sensor in the detection of antibiotics from complex matrix samples. The introduced strategy can easily be extended to the detection of other antibiotics and harmful substances by screening for suitable MIPs. However, the binding volume and structural rigidity of MIP in the sensor still requires further improvement.

\section{Conflicts of interest}

There are no conflicts to declare.

\section{Acknowledgements}

This project was supported by the Natural Science Foundation of Hainan Province of China (No. 217222) and the Central Public-interest Scientific Institution Basal Research Fund for
Chinese Academy of Tropical Agricultural Sciences (No. 1630082017002 and 1630082017003$)$.

\section{Notes and references}

1 B. J. Lemos, F. G. Castro, L. S. Santos, B. P. Mendonça, V. R. Couto and J. J. Fernandes, J. Anim. Sci., 2016, 94, 4307.

2 C. A. Hajee, H. J. van Rhijn, J. J. Lasaroms, H. J. Keukens and J. J. De, Analyst, 2001, 126, 1332.

3 J. Boison, S. Lee and R. Gedir, J. AOAC Int., 2009, 92, 329.

4 P. Gupta and R. N. Goyal, RSC Adv., 2015, 5, 40444.

5 J. Hou, H. Li, L. Wang, P. Zhang, T. Zhou, H. Ding and L. Ding, Talanta, 2016, 146, 34.

6 J. Wackerlig and R. Schirhagl, Anal. Chem., 2016, 88, 250.

7 K. Haupt and K. Mosbach, Chem. Rev., 2000, 100, 249.

8 S. Li, C. Liu, G. Yin, Q. Zhang, J. Luo and N. Wu, Biosens. Bioelectron., 2017, 91, 687.

9 A. N. Kawde, N. Baig and M. Sajid, RSC Adv., 2016, 6, 91325. 10 Y. Wang, M. Han, X. Ye, K. Wu, T. Wu and C. Li, Microchim. Acta, 2017, 184, 195.

11 J. R. Wei, Y. L. Ni, W. Zhang, Z. Q. Zhang and J. Zhang, Anal. Chim. Acta, 2017, 960, 110.

12 M. Mehrzad-Samarin, F. Faridbod, A. S. Dezfuli and M. R. Ganjali, Biosens. Bioelectron., 2017, 92, 618.

13 S. Wagner, J. Bell, M. Biyikal, K. Gawlitza and K. Rurack, Biosens. Bioelectron., 2018, 99, 244.

14 W. Han, L. Gao, X. Li, L. Wang, Y. Yan, G. Che, B. Hu, X. Lin and M. Song, RSC Adv., 2016, 84, 81346.

15 J. Hou, H. Li, L. Wang, P. Zhang, T. Zhou, H. Ding and L. Ding, Talanta, 2016, 146, 34.

16 F. H. Koppens, T. Mueller, P. Avouris, A. C. Ferrari, M. S. Vitiello and M. Polini, Nat. Nanotechnol., 2014, 9, 780.

17 F. Shi, Y. Zhang, W. Na, X. Zhang, Y. Li and X. Su, J. Mater. Chem. B, 2016, 4, 3278.

18 A. M. Şenol, Y. Onganer and K. Meral, Sens. Actuators, B, 2017, 239, 343-351.

19 Y. P. Sun, B. Zhou, Y. Lin, W. Wang, K. A. Fernando, P. Pathak, M. J. Meziani, B. A. Harruff, X. Wang, H. F. Wang, P. G. Luo, H. Yang, M. E. Kose, B. L. Chen, L. M. Veca and S. Y. Xie, J. Am. Chem. Soc., 2006, 128, 7756.

20 Y. M. Guo, Z. Wang, H. W. Shao and X. Y. Jiang, Carbon, 2013, 52, 583.

21 Z. Liu, P. Yin, H. Gong, P. Li, X. Wang and Y. He, J. Lumin., 2012, 132, 2484. 